\title{
Exercice du droit de chasse et droit de propriété
}

Exercise of hunting right and property right

\section{Maître Alix Domas-Descos}

\section{(2) OpenEdition}

\section{Journals}

Édition électronique

URL : http://journals.openedition.org/economierurale/3382

DOI : 10.4000/economierurale.3382

ISSN : 2105-2581

Éditeur

Société Française d'Économie Rurale (SFER)

Édition imprimée

Date de publication : 1 mars 2012

Pagination : 114-125

ISSN : 0013-0559

Référence électronique

Maître Alix Domas-Descos, «Exercice du droit de chasse et droit de propriété », Économie rurale [En ligne], 327-328 | janvier-mars 2012, mis en ligne le 01 mars 2014, consulté le 30 avril 2019. URL: http://journals.openedition.org/economierurale/3382; DOI : 10.4000/economierurale.3382 


\section{Exercice du droit de chasse et droit de propriété}

Maître Alix DOMAS-DESCOS - avocat au Barreau de Paris

a.domas-descos@cabinet-chavanes.com

D roit à valeur constitutionnelle, consacré par les articles 2 et 17 de la Déclaration des droits de l'homme et du citoyen, le droit de propriété est un droit fondamental, qui s'exprime notamment au travers de la libre disposition de ses biens par un propriétaire, et du droit de chacun au respect de ses biens ${ }^{1}$.

Attribut du droit de propriété, le droit de chasse appartient au propriétaire du fonds, qui peut se le réserver, le donner en location à un tiers ou le louer au preneur de l'immeuble rural, en même temps que le bien sûr lequel il s'exerce, ou le confier à une Association communale de chasse agréée (ACCA).

L'exercice de la chasse et du droit de chasse est perçu tantôt comme une limite à la libre disposition des biens, voire une atteinte au respect même de la propriété, ce sera la première partie de cet article, tantôt comme un élément de valorisation et de protection de celle-ci exprimé dans la seconde partie.

Avec la création du système particulier des ACCA, le droit de propriété et de disposer librement de ses biens, s'est trouvé restreint au bénéfice du droit de chasse. Ces dispositions ont été contestées tout d'abord par des propriétaires hostiles à la chasse, revendiquant la reconnaissance, comme corollaire à leur droit de propriété, du droit d'opposition ou de non chasse. Ce système d'organisation de la chasse a également été critiqué par des propriétaires chasseurs, désireux de recouvrer le plein exercice de

1. Conseil constitutionnel 16 janvier 1982, Cour administrative d'appel de Bordeaux, 14 juin 2005, $\mathrm{n}^{\circ}$ 01BX02337, ACCA de Bignoux et $\mathrm{n}^{\circ}$ 01BX01733, ACCA de Liguge. leurs droits de chasse soustraits au bénéfice des associations. En outre, les rapports entre droit de propriété et droit de chasse se traduisent aussi au travers d'autres conflits d'usage, comme par exemple, en matière de circulation des véhicules à moteur.

Néanmoins, le droit de chasse et son exercice peuvent être des éléments de valorisation des domaines ruraux et forestiers. En effet, le droit de chasse peut être loué dans le cadre d'un bail rural ou d'un bail de chasse, qui confère au preneur des obligations quant à la pratique de la chasse et de son organisation. À cette occasion, cet exercice peut interférer avec le respect d'autres réglementations (comme par exemple, l'accueil du public au sein du domaine de chasse) ou d'autres activités (loisirs, sylviculture, exploitation touristique ou agricole etc.).

\section{Les conflits entre droit de propriété et droit de chasse}

L'exercice du droit de chasse en France s'est organisé, depuis la Révolution française, autour du foncier et du consentement du propriétaire. Deux conceptions se sont opposées à cet égard en 1789 : l'une, soutenue par Mirabeau, liait droit de chasse et propriété, l'autre, soutenue par Robespierre, prônait une liberté de chasser en tous lieux, au bénéfice de tous citoyens et sans conditions. La formule actuelle de l'article L. 422-1 du Code de l'environnement : «Nul n'a la faculté de chasser sur le terrain d'autrui sans le consentement du propriétaire ou de ses ayants-droits », est un héritage direct des lois du 3 mai 1844 et de la loi du 30 avril 1790, dont s'inspiraient les 
anciens articles L. $222-1$ et 365 du Code rural, concernant l'exercice de la chasse.

Tout en protégeant les terrains d'un propriétaire contre une pratique irrégulière ou non autorisée de la chasse, il a été apporté des limites à l'usage du droit de propriété, afin de garantir l'exercice du droit de chasse par le plus grand nombre et dans des conditions satisfaisantes, notamment eu égard à la sécurité des biens et des personnes.

En effet, le droit de propriété, malgré sa reconnaissance constitutionnelle, n'est pas un droit défendu de manière aussi absolue que d'autres libertés fondamentales, et subit, en conséquence, certaines restrictions ou limites (Vigier, 2007).

Afin de répondre notamment au morcellement parcellaire des territoires de chasse, au développement de la chasse dite banale (la pratique de la chasse est admise tant que le propriétaire n'a pas expressément manifesté son intention de la réserver), et de favoriser l'accès du plus grand nombre à un territoire de chasse plus important, le législateur a mis en place en 1964 (au travers de la dite "loi Verdeille »), le système d'une organisation collective de la chasse, avec la création des ACCA, désormais codifié aux articles L. 422-2 et suivants du Code de l'environnement.

Ces associations «ont pour but d'assurer une bonne organisation technique de la chasse. Elles favorisent sur leur territoire le développement du gibier et de la faune sauvage dans le respect d'un véritable équilibre agro-sylvo-cynégétique, l'éducation cynégétique de leurs membres, la régulation des animaux nuisibles et veillent au respect des plans de chasse en y affectant les ressources appropriées. Elles ont également pour objet d'apporter la contribution des chasseurs à la conservation des habitats naturels, de la faune et de la flore sauvages »(article L. 422-2 dudit Code).

Ce système a généré de nombreux contentieux, illustrant l'ambiguïté des rapports entre droit de propriété et droit de chasse (Peignot 2006 ; Lagier, 2008). L'activité de ces associations s'exerce, en effet, dans le respect des propriétés, des cultures et des récoltes (article L. 422-2 dernier alinéa du Code de l'environnement).

Les contestations du dispositif des ACCA sont principalement venues de propriétaires, hostiles à la chasse et refusant l'inclusion de leurs terrains dans le territoire soumis à l'action des ACCA, mais aussi de propriétaires, chasseurs ou non opposant à la chasse, désireux de récupérer les droits de chasse attachés à leurs terrains et soustraits au bénéfice de l'ACCA.

\section{La reconnaissance d'un droit de non chasse, corollaire du droit de propriété}

Certains propriétaires, dont les convictions personnelles s'opposaient à la chasse, ont considéré que l'apport forcé de leurs droits de chasse à une ACCA, constituait une privation anormale de leur droit d'usage sur leurs fonds, tel que défini et garanti à l'article $1 \mathrm{du}$ Protocole additionnel de la Convention européenne de sauvegarde des droits de l'homme et des libertés fondamentales, ceux-ci devant subir la présence de chasseurs sur leurs propriétés et ne pouvant affecter leurs terrains à un usage conforme à leurs convictions personnelles (comme la création d'une réserve naturelle par exemple).

Ils considéraient également que l'adhésion forcée à l'ACCA qui en découlait, au mépris de leurs positions éthiques, restreignait leur liberté d'association, protégée à l'article 11 de ladite Convention.

Aux termes du célèbre arrêt Chassagnou, du 29 avril 1999², la Cour européenne des droits de l'homme a sanctionné le dispositif français instauré par la loi Verdeille, et reconnu notamment que :

- l'apport forcé à une ACCA des droits de chasse sur leurs terrains par les propriétaires constituait une ingérence dans leur jouissance des droits d'usage sur leurs

2. Cour européenne des droits de l'homme, 29 avril 1999, Arrêt CHASSAGNOU et autres c/France, requêtes $n^{\circ} 25088 / 94,28331 / 95$ et 28443/95 
biens, que les requérants tenaient directement de leur qualité de propriétaire, au mépris des dispositions du second alinéa de l'article $1 \mathrm{du}$ Protocole additionnel (point 74 de l'arrêt précité) ;

- malgré le caractère d'intérêt général que revêtaient les objectifs de la loi Verdeille, en évitant une pratique anarchique de la chasse et en favorisant une gestion rationnelle du patrimoine cynégétique, le système français plaçait les requérants dans une situation qui rompait «le juste équilibre devant régner entre la sauvegarde du droit de propriété et les exigences d'intérêt général » (point 79 dudit arrêt) ;

- dès lors, l'obligation d'apports de leurs droits de chasse par les petits propriétaires, "pour que des tiers en fasse un usage totalement contraire à leurs convictions » constituait une charge démesurée et une discrimination non fondée qui devait être sanctionnée au double visa de l'article $1 \mathrm{du}$ Protocole additionnel et de l'article 14 de la Convention, relatif aux discriminations fondées sur la fortune, l'opinion et le mode vie.

Concernant l'atteinte à la liberté d'association, la Cour a considéré que ce système d'adhésion et d'apport « forcés » aux ACCA ne pouvait être considéré comme proportionné au but poursuivi, et allait au-delà de ce qui était nécessaire pour assurer un juste équilibre entre des intérêts contradictoires.

À la suite de cet arrêt, le droit de propriété s'est ainsi vu attribuer un nouveau corollaire, le droit de non chasse ou droit d'opposition, au bénéfice des propriétaires dont les convictions personnelles s'opposent à la chasse (Peignot, op. cit.).

Cet arrêt a également contraint le législateur français à mettre son dispositif d'organisation des droits de chasse en conformité avec la position de la jurisprudence européenne, avec les modifications apportées en $2000^{3}$ (Patriat, 2000).

3. Loi n ${ }^{\circ} 2000-698$ du 26 juillet 2000.
Les propriétaires, opposants à la chasse, sont désormais libérés de l'obligation d'apports de leurs droits de chasse à l'ACCA et de l'obligation d'adhérer à une telle association.

Néanmoins, la reconnaissance de ce droit de non chasse n'a pas mis fin au système français d'organisation de la chasse dans le cadre des ACCA (Privat, 2007). De même que le droit de chasse du propriétaire, attribut du droit de propriété, n'est pas absolu, le droit de non chasse est encadré.

\section{Une application encadrée du droit de non chasse}

Comme l'a rappelé le Conseil d'État dans un arrêt du 9 novembre $2007^{4}$, « les dispositions $d u$ Code de l'environnement relatives aux associations communales de chasse agréées ont pour objet de concilier l'organisation $d u$ contrôle des espèces, qui implique que les territoires soumis à l'action des associations de chasse agréées ne puissent être réduits de façon immédiate et imprévisible à la seule initiative des propriétaires concernés et le droit de ceux-ci de s'opposer, en raison de leurs convictions personnelles, à la pratique de la chasse sur leurs terrains ».

L'application du droit de non chasse s'accompagne de certaines conditions, édictées notamment aux articles L. 422-14 et L. 422$15 \mathrm{du}$ Code de l'environnement.

Tout d'abord, l'opposition du propriétaire, visée à l'article L. 422-10 5 ${ }^{\mathrm{e}} \mathrm{du}$ Code de l'environnement, n'est recevable que si elle porte sur l'ensemble des terrains appartenant aux propriétaires ou copropriétaires en cause. Elle vaut renonciation à l'exercice du droit de chasse sur ces terrains.

Toutefois, le droit de chasser du preneur d'un immeuble rural n'est pas concerné par cette opposition à la chasse (article L. 415$7 \mathrm{du}$ Code rural et de la pêche maritime). Dans ce cas, le droit de chasser du preneur subit les mêmes restrictions que celles

4. Conseil d'État, 9 novembre 2007, n 296858. 
ressortissant des usages locaux qui s'appliquent sur les territoires de chasse voisins et celles résultant du schéma départemental de gestion cynégétique.

Ensuite, le propriétaire est tenu de procéder à la signalisation de son terrain matérialisant l'interdiction de chasser. Il est également tenu, sous peine de voire sa responsabilité délictuelle engagée, de procéder ou de faire procéder à la destruction des animaux nuisibles et à la régulation des espèces présentes sur son fonds et qui causent des dégâts.

Par ailleurs, le nouveau propriétaire, en cas de vente des terrains bénéficiant d'une opposition, peut maintenir cette opposition à raison de ses convictions personnelles, dans un délai de six mois courant à compter du changement de propriétaire. À défaut, ces terrains sont intégrés dans le territoire de l'association (article L. 422-19 du Code de l'environnement).

Ces dispositions sont parfois considérées par certains propriétaires comme contraignantes et portant atteinte à leurs droits de propriété et de non chasse.

Cependant, le Conseil d'État ${ }^{5}$ a jugé, dans une décision du 9 novembre 2007 $\left(n^{\circ} 296858\right)$, qu'elles ne constituent pas une atteinte disproportionnée au droit de propriété, ni à la liberté d'association, par rapport au but d'intérêt général poursuivi, et ne méconnaissent pas, en conséquence l'article 1 du Protocole additionnel à la Convention européenne des droits de l'homme et des libertés fondamentales relatif à la propriété, ni l'article 11 de ladite Convention relatif à la liberté d'association.

En outre, le Conseil d'État ${ }^{6}$ a clairement rappelé, dans une autre décision du même jour ( $\left.\mathrm{n}^{\circ} 293644\right)$ que les dispositions de l'article L. 422-10 5 et L. 422-14 du Code de l'environnement, relatives au droit d'opposition des propriétaires, n'ont pas pour effet de priver les propriétaires de leur droit

5. Conseil d'État, 9 novembre 2007, $\mathrm{n}^{\circ} 296858$.

6. Conseil d'État, 9 novembre 2007, n 293644. de propriété, mais seulement d'apporter des limitations à leur droit d'usage, lesquelles ne sont pas disproportionnées au regard du but légitime poursuivi par la législation relative aux ACCA, tendant à assurer une bonne organisation technique de la pratique de la chasse et le respect de l'équilibre agrosylvo-cynégétique.

\section{Un droit de chasse lui aussi encadré}

L'opposition à l'apport forcé des droits de chasse aux ACCA est également venue de propriétaires, chasseurs, ou du moins, non opposés à la pratique de chasse, qui souhaitaient recouvrer le bénéfice de leurs droits de chasse soustraits au bénéfice de l'ACCA et les conserver pour eux-mêmes. La loi Verdeille a été perçue par certains propriétaires chasseurs, comme une atteinte, voire une suppression, de leur droit de chasse.

Certes, l'article L. 422-10-3 ${ }^{\mathrm{e}}$ du Code de l'environnement permet au propriétaire de terrains d'une certaine superficie de conserver le droit de chasse attaché à ses terrains, pour son propre usage, sans permettre aux membres de l'ACCA d'en bénéficier, mais pour les petits propriétaires, dont la superficie est inférieure à ce seuil, la soumission à l'ACCA est obligatoire.

La Cour européenne des droits de l'homme, dans un arrêt du 6 décembre $2007^{7}$, qui reprend d'ailleurs bon nombre des considérants de l'arrêt Chassagnou ci-avant visé et en précise les conséquences, et le Conseil d'État, aux termes de deux arrêts du 16 juin $2008^{8}$, ont précisé et rappelé les conditions du droit d'opposition à la soumission aux ACCA et de retrait des terrains soumis à l'action de l'association (Lagier, op. cit. ; De Montbron 2008).

7. Cour européenne des droits de l'homme, 6 décembre 2007, Arrêt M. Mme Gilbert Baudinière c/ France, requête $\mathrm{n}^{\circ} 25708 / 03$ et Arrêt M. Mme René Vauzelle c/ France, requête $n^{\circ} 25719 / 03$ 8. Conseil d'État, 16 juin 2008, M. H. n 296632 ; Conseil d'État, 16 juin 2008, Association communale de chasse agréée de Louin, n 297568. 
En effet, le propriétaire d'une superficie totale inférieure à la valeur requise pour s'opposer à la soumission des terrains à l'action de l'ACCA, sur le fondement de l' article L. 422-10-3 $3^{\mathrm{e}}$ du Code de l'environnement, dispose de deux possibilités :

- soit il renonce à son droit de chasse et invoque des convictions personnelles opposées à la pratique de la chasse (L.422-10, 5 ${ }^{\mathrm{e}}$, du Code de l'environnement) ;

- soit, à défaut de justifier de telles positions personnelles, il apporte ses droits à l'ACCA et bénéfice ainsi de l'accès à l'ensemble du territoire de l'association et à de meilleures conditions de chasse.

Comme le souligne la jurisprudence, cette différence de traitement, objective et raisonnable, entre petits et grands propriétaires est instituée dans l'intérêt des chasseurs propriétaires de petites parcelles, qui peuvent ainsi se regrouper et bénéficier d'un territoire de chasse plus grand. En tout état de cause, ces propriétaires disposent toujours de la possibilité d'affecter leur terrain à un usage conforme à leurs convictions personnelles.

Il n'y a donc pas atteinte au droit de propriété et de libre usage d'un fonds par son propriétaire.

Désormais, la validité du système français d'organisation de la chasse au travers des ACCA, semble entérinée, tant au regard de l'instauration et de l'application du droit de non chasse, corollaire du droit de propriété, que de la protection du droit de chasse, attribut du droit de propriété.

Un équilibre, encore précaire peut-être, compte tenu de l'existence de multiples problématiques soulevées par leurs usages, semble s'être instauré autour du droit de propriété et de ses attributs, droits de chasse et d'opposition.

Les rapports entre droit de chasse et droit de propriété s'expriment également lors de la pratique même de la chasse. Il n'est pas question ici de dresser une liste exhaustive des multiples problématiques engendrées, mais de présenter des exemples concrets de conflits d'usage, notamment concernant la sécurité des biens et des personnes.

\section{Conflits d'usage La sécurité des biens et des personnes}

\section{L'exemple de la circulation \\ des véhicules à moteur}

Indépendamment du pouvoir dont dispose tout propriétaire pour limiter l'accès à sa propriété, la circulation des véhicules terrestres à moteur est, sauf exception, interdite par la loi dans les espaces naturels et le milieu forestier, sous peine d'amendes et de mise en fourrière?

Certaines activités motorisées, comme les motoneiges, font également l'objet de réglementations particulières et certains espaces naturels bénéficient d'une protection renforcée du point de vue environnemental (charte de territoire, directives montagnes etc.).

La notion d'ouverture d'un espace privé à la circulation publique est soumise à l'appréciation souveraine des juges du fond. Une voie est généralement présumée ouverte dès lors qu'elle est manifestement praticable par un véhicule de tourisme non spécialement adapté au tout - terrain. Les simples sentiers ou layons ne sont pas soumis à signalisation car ils sont présumés fermés à la circulation de par leurs seules caractéristiques $^{10}$.

Néanmoins, l'accord du propriétaire est un préalable indispensable à l'utilisation de la voie. Celui-ci peut aussi prendre la décision de fermer la voie à la circulation des

9. Articles L. 362-1 et suivants du Code de l'environnement ; articles L. 321-1 et suivants, L.331-2 et suivants, R. 322-1 et suivants, R. 331-3 et suivants et R. 412-16 du Code forestier ; Article R. 411-24 du Code de la route.

10. Cour de cassation, Chambre criminelle, 19 février 1957 ; Cour d'appel de Chambéry, Chambre correctionnelle, 29 mars 1995 ; Cour de cassation, Chambre criminelle, 18 février 2003. 
véhicules à moteur. La fermeture d'une voie peut aussi résulter d'une mesure de police du maire ou du préfet en vertu de son pouvoir en matière de police, de circulation et de stationnement ${ }^{11}$.

Le propriétaire, sous peine de voir sa responsabilité engagée, ne pourra, cependant, utiliser n'importe quel dispositif physique comme des câbles, des chaînes, des barrières, sans des précautions évidentes de signalisation et d'identification de l'obstacle à la pénétration sur la voie ${ }^{12}$. La responsabilité civile de l'exploitant forestier peut en effet être recherchée sur le fondement de l'article 1384 du Code civil, en raison d'un fil de fer tendu au travers d'un chemin et heurté par un motocycliste ${ }^{13}$ ou en cas de dommages causés par un arbre $^{14}$ par exemple.

Par conséquent, il y a lieu, préalablement à la pratique de la chasse, pour éviter tout conflit d'usage entre l'exercice de l'activité de chasse et le respect de ses biens par un propriétaire, ainsi que la mise en jeu de responsabilités pénale et civile qui peuvent en découler, de vérifier les conditions de circulation dans le territoire sur lequel se déroule la chasse.

Si le droit de propriété et le droit de chasse peuvent dans leur exercice respectif s'opposer ou créer des conflits d'usage, le droit de chasse peut se révéler un élément de valorisation des biens.

11. Articles L. 2213-4, L. 2215-3 et L. 2212-4 du Code général des collectivités territoriales.

12. La circulaire $n^{\circ}$ DGA/SDAJ/BDEDP $n^{\circ} 1 \mathrm{du}$ ministère de l'Écologie du 6 septembre 2005 conseille "vivement de matérialiser la fermeture de la voie sur le terrain ", et recommande même la pose d'un panneau B.O. ou d'un dispositif de fermeture (barrières, plots etc.).

13. Cour de cassation, deuxième chambre civile, 30 novembre 1994.

14. Cour de cassation, 30 avril 1952 ; Cour de Cassation, deuxième chambre civile, 29 mars 1971, Cour de Cassation, deuxième chambre civile, 10 février 1985 ; Cour d'appel de Dijon, 5 avril 1996.

\section{Protection et valorisation d'un bien par l'exercice et l'exploitation du droit de chasse}

Si le droit de chasse, au travers de son exercice, est souvent perçu comme une entrave à la libre disposition de ses biens par un propriétaire, il s'avère également être un élément de valorisation et d'exploitation du bien concerné. Attribut du droit de propriété, le droit de chasse appartient au propriétaire du fonds qui peut se le réserver, le donner en location à un tiers ou au preneur de l'immeuble rural ou encore le conférer à l'ACCA.

Néanmoins, cette mise en valeur interfère tant avec l'exploitation d'autres activités (agricole, forestière, touristique, etc.) et se trouve régie par d'autres réglementations, d'ordre urbanistique ou économique, plus ou moins contraignantes, tant quant à la libre disposition ou le libre usage de ses biens par le propriétaire, qu'à la pratique même de la chasse.

D'autres mécanismes accordent également une indemnisation au propriétaire d'un bien ayant subi des dommages par le gibier.

\section{Droit de chasse, droit de chasser et baux ruraux}

Si le bailleur propriétaire, dans le cadre d'un bail rural, dispose du droit de chasse sur ses propriétés, le preneur, titulaire du bail, bénéficie du droit de chasser, en vertu des dispositions de l'article L. 415-7 du Code rural et de la pêche maritime (Minne, 2007). Est réputée non écrite toute clause stipulant que le preneur ne dispose pas du droit de chasser sur les terrains loués (article L. 415-12 du Code rural et de la pêche maritime).

Le droit de chasser du preneur est un droit personnel, attaché à la qualité de preneur d'un bail rural : celui-ci ne peut en effet en faire bénéficier un tiers ou un membre de sa famille ${ }^{15}$. En cas d'association

15. Cour de cassation, chambre sociale, 13 avril 1967. 
au bail rural d'un descendant par exemple, le droit de chasser bénéficiera aux copreneurs.

L'exercice par le preneur de son droit de chasser, concurremment à celui du droit de chasse du propriétaire du fonds loué, ne prive pas le preneur de demander au bailleur, ou au détenteur du droit de chasse si celuici a fait l'objet d'un bail particulier par le bailleur, réparation des dommages causés par le gibier (article D. 415-3 du Code rural et de la pêche maritime). En outre, le bailleur ne peut réclamer au preneur aucune majoration du prix du bail en raison de l'exercice $\mathrm{du}$ droit de chasser (article D. 415-6 du Code rural et de la pêche maritime).

L'exercice du droit de chasser est par ailleurs subordonné à l'observation et au respect des dispositions légales et réglementaire concernant la chasse. Ainsi, le droit de chasser ne peut s'exercer dans les réserves cynégétiques autorisées, ni porter sur le gibier d'élevage (article D. 415-5 du Code rural et de la pêche maritime).

En outre, lorsque le bailleur, ou le détenteur du droit de chasse, s'impose des restrictions pour la protection du gibier ou l'amélioration des conditions de chasse, par exemple quant au nombre de jours de chasse, espèces etc., celles-ci doivent être communiquées au preneur et s'imposent également à lui, sauf décision contraire du tribunal paritaire des baux ruraux.

Le propriétaire du fonds peut également avoir formé opposition à la chasse en application de l'article L. 422-10 du Code de l'environnement ${ }^{16}$. Dès lors, le droit de chasser subit les mêmes restrictions que celles ressortissant des usages locaux qui s'appliquent sur les territoires de chasse voisins et celles résultant du schéma départemental de gestion cynégétique (articles L. 421-7 du Code rural et de la pêche maritime et L.422-14 du Code de l'environnement).

16. Sur le droit de non chasse, $c f$. infra.
Lorsque le propriétaire bailleur a fait apport de son droit de chasse à une ACCA, le preneur en devient membre et bénéficie alors du droit de chasser sur tout le territoire de l'association ${ }^{17}$.

Le droit de chasse du propriétaire du fonds peut également être cédé ou loué ; dès lors, le droit de chasse du propriétaire et le droit de chasser du preneur à bail rural s'exercera concurremment avec le détenteur du droit de chasse.

Il conviendra toutefois, aux termes du bail de chasse concédé, de préciser les conditions d'exercice de la chasse et notamment l'existence d'un bail rural, d'une autre activité (exploitation forestière, minière, etc.) ou d'éventuelles restrictions à sa pratique, pour un exercice harmonieux des droits de chacun et prévenir tout conflit d'usage.

\section{2. Établissement des baux de chasse Entre intérêts du propriétaire et modalités de chasse}

L'établissement de baux de chasse peut se faire par négociation de gré à gré, mais également par voie d'adjudication, selon un cahier des charges prédéfini (établissement d'un plan de chasse etc.).

Le bail de chasse est distinct du bail rural et de son régime légal fort protecteur. Non soumis au statut des baux ruraux (article L. 415-10 du Code rural et de la pêche maritime), le bail de chasse a un caractère civil, qu'il convient de rappeler explicitement aux termes de la convention pour éviter - ou limiter du moins - tout risque de requalification. En cas de présence de bâtiments loués dans le cadre de la chasse, tels qu'abris, granges, maisons etc., il est prudent de spécifier que le régime des baux d'habitation pour ces bâtiments est expressément exclu et limite ainsi les demandes de requa-

17. Article L. 422-21-1 du Code de l'environnement. Cette disposition s'inscrit évidemment dans la logique du système des ACCA, tendant à améliorer les conditions de chasse et à favoriser l'exercice de la chasse par le plus grand nombre. 
lification et le maintien dans les lieux du locataire de la chasse à ce titre. De même, il convient de régler les modalités d'occupation et d'entretien de ces éléments, pour éviter tout contentieux lors du bail comme à la libération des lieux loués.

Outre les clauses habituelles d'un bail (description et destination du bien loué, durée, loyer, modalités de règlement, charges, etc.), doivent notamment être définis avec précision, aux termes du bail de chasse, les points suivants :

- les personnes bénéficiant du droit de chasse : preneur du bail de chasse, conjoints, ascendants, invités...

- le nombre de chasseurs autorisés à chasser :

- l'établissement du plan de chasse et la présentation de celui-ci au bailleur;

- les règles de sécurité sur l'organisation et la signalisation de la pratique de la chasse, (panneautage de la zone de chasse, information du public, circulation des véhicules, accès des secours, dispositif d'alerte, cohabitation avec une autre activité, exploitation forestière ou bail rural par exemple...), et, le cas échéant, les sanctions en cas d'inobservations de ces règles, comme l'avertissement par le bailleur, l'arrêt de l'action de chasse, voire la résiliation du bail de chasse ;

- les clauses relatives à la responsabilité du bailleur et du locataire de chasse, en particulier quant aux dommages causés à des tiers, ou aux biens du propriétaire des lieux à l'occasion de l'exercice du droit de chasse ;

- les clauses relatives à l'entretien du milieu physique (allées, chemins forestiers, étangs, etc.).

Par ailleurs, l'action du preneur devra s'inscrire dans le respect des réglementations et normes en vigueur, telles que les prescriptions en matière de protection des milieux naturels, d'urbanisme, relatives par exemple à la constructibilité ou non, à l'aménagement ou à la rénovation de bâtiment, ou encore à l'accueil du public lors des campagnes de chasse.

\section{Interaction entre activité de chasse et réglementation}

\section{L'exemple des établissements}

recevant $d u$ public

La réglementation dite des Établissements recevant du public (ERP), visée aux articles R. 123-2 et suivants du Code de la construction et de l'habitation, peut trouver à s'appliquer en matière de bâtiments dévolus à l'activité de chasse. En effet, constituent des établissements recevant du public tous bâtiments, locaux et enceintes dans lesquels des personnes sont admises, soit librement, soit moyennant une rétribution ou une participation quelconque, ou dans lesquels sont tenues des réunions ouvertes à tout venant ou sur invitation, payantes ou non. Sont considérées comme faisant partie du public toutes les personnes admises dans l'établissement à quelque titre que ce soit en plus du personnel.

Ces établissements sont soumis à une réglementation stricte, fonction de la catégorie dans laquelle ils se trouvent ${ }^{18}$, notamment concernant leur aménagement et sécurité et exposent leur propriétaire et exploitants à des obligations strictes.

Les prescriptions sur les mesures de sécurité, d'aménagement, ou d'accessibilité, devront alors être respectées par le preneur. Le bailleur devra exiger leurs observations et leurs mises en œuvre, dans le cadre de l'exercice du bail de chasse. À défaut de satisfaire à ces exigences, la responsabilité du locataire pourra être recherchée et le bail résilié.

Cette situation peut s'avérer contraignante pour le preneur du bail de chasse, comme pour le propriétaire, mais ces dispositions

18. L'article R.123-19 du Code de la construction et de l'habitation dispose que les catégories d'ERP sont établies en fonction de l'effectif du public et du personnel qui le fréquente. Les obligations seront plus ou moins contraignantes suivant la catégorie. 
visent à garantir un exercice contrôlé et en toute sécurité de la chasse. En outre, elles contribuent à renforcer la valeur patrimoniale de la chasse louée, même si elles nécessitent un investissement financier souvent important.

D'autres mécanismes, prévus au titre de la pratique de la chasse, permettent plus indirectement d'assurer une protection de la valeur du fonds.

\section{La protection d'un bien foncier par le bénéficiaire du droit de chasse ou de sa pratique}

\section{L'exemple de l'indemnisation des dégâts}

- La responsabilité du propriétaire du fonds dont le gibier cause des dommages au fonds voisin peut être engagée sur le fondement de la responsabilité délictuelle ${ }^{19}$. Lorsque celuici a loué ou cédé son droit de chasse à un tiers, à titre onéreux ou gratuit, la responsabilité du propriétaire est transférée au locataire ou cessionnaire, qui devra prendre toutes les mesures utiles pour que les terrains voisins ne subissent pas de dommages dus au gibier. À noter cependant que le propriétaire demeure toutefois responsable lorsque la faute servant de base à la demande lui incombe exclusivement ${ }^{20}$.

La jurisprudence exige de prouver non seulement que le gibier s'est développé sur le fonds du propriétaire en cause, de manière excessive, et que les animaux proviennent bien du terrain de ce dernier, ce qui est parfois difficile en cas d'animaux nomades ou errants ${ }^{21}$, mais également que les dégâts causés sont la conséquence directe de la négligence, de l'imprudence ou de la faute du propriétaire ${ }^{22}$. Ce sont généralement l'in-

19. Article 1382 et 1383 du Code civil.

20. Tribunal civil de Melun, 21 février 1862

21. Cour de cassation, deuxième chambre civile,

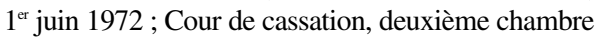
civile, 19 mars 1997, n ${ }^{\circ}$ 95-14.677 ; Cour de cassation, deuxième chambre civile, 28 mars 1990, $n^{\circ}$ 89-12.646. 22. Cour d'appel de Paris, 13 décembre 2004, $n^{\circ} 03-$ 16.881 ; Cour de cassation, deuxième chambre civile, 5 juin 1964, 14 octobre 1992. suffisance des mesures de destruction du gibier prises par le propriétaire ou une protection excessive des animaux par celui-ci qui sont à l'origine du pullulement litigieux ${ }^{23}$.

Pour apprécier le caractère excessif, le juge retient des critères quantitatifs, tels que le nombre ou l'importance des coulées, trous, terriers ou déjections, le nombre de têtes de cervidés par rapport à la superficie ${ }^{24}$; il peut également ordonner le recours à l'expertise.

Concernant la réparation des dommages, quel que soit la valeur de la demande, ces litiges sont de la compétence du tribunal d'instance (article R. 426-21 du Code de l'environnement) ; les recours pour excès de pouvoir contre certaines décisions administratives relatives aux modalités d'exercice de la procédure d'indemnisation sont de la compétence de la juridiction administrative, par exemple pour contestation des barèmes d'indemnisation ${ }^{25}$.

Les propriétaires dont les cultures ou les récoltes ont subi des dommages causés par des sangliers ou du grand gibier provenant d'une réserve ou d'un fonds sur lequel est exécuté un plan de chasse, peuvent demander une indemnisation auprès des pouvoirs publics. La procédure en indemnisation est définie et régie par les articles L. 426-1 et suivants du Code de l'environnement, mais n'exclut pas une action fondée sur l'article 1382 du Code civil (responsabilité pour faute).

- Le bénéficiaire du droit de chasse

Il a également l'obligation, en application de l'article L. 425-12 du Code de l'environnement ${ }^{26}$, d'indemniser le

23. Cour de cassation, civile, 4 janvier 1974 ; Cour de cassation, deuxième chambre civile, 21 juillet 1992, n $91-13.823$, Cour de cassation, deuxième chambre civile, 18 septembre 2003, $\mathrm{n}^{\circ}$ 02-14.638 24. Cour de cassation, deuxième chambre civile, 15 décembre 1975.

25. Tribunal des conflits, 3 mai 1999, $\mathrm{n}^{\circ} 3114$. 26. Les règles d'indemnisation des dégâts sylvicoles causés par le grand gibier ont été précisées par le décret $n^{\circ} 2008-259$ du 14 mars 2008 . 
propriétaire d'un fonds des dégâts causés par le gibier lorsque le nombre minimum d'animaux attribués par le plan de chasse n'a pas été prélevé. Par ailleurs, les propriétaires de territoires forestiers gérés conformément à l'article L. 4 du Code forestier ${ }^{27}$ et dont les terrains sont incorporés dans le territoire de chasse d'une ACCA, peuvent demander réparation aux bénéficiaires de droit de chasse des dommages causés par le grand gibier aux plants et semis ou peuplements adultes, dans la limite d'un plafond d'indemnité calculé à l'hectare par le préfet du département et d'un barème fixé conjointement par les ministres chargés de la chasse et de la forêt $t^{28}$.

Ces dispositions sont applicables lorsque l'équilibre sylvo-cynégétique est fortement perturbé, c'est-à-dire dès lors que les dégâts causés compromettent la régénération des peuplements forestiers, qui s'apprécie au regard du nombre ou de la densité des tiges ou de plants viables, inférieurs ou non à un seuil fixé par le préfet de région.
En outre, les propriétaires forestiers peuvent prendre des mesures destinées à renforcer la protection des régénérations des peuplements forestiers et demander au bénéficiaire du droit de chasse, tout ou une partie des dépenses de protection ainsi engagées.

$$
\text { * }
$$

Droit de chasse et droit de propriété entretiennent des relations ambiguës, tantôt conflictuelles, lorsqu'il s'agit de restreindre l'exercice d'un droit par rapport à l'autre, tantôt complémentaires, lorsque leur mise en jeu concertée favorise l'exercice de la chasse et la valorisation de la propriété foncière ou forestière. $\mathrm{La}$ recherche d'un équilibre entre chasse et propriété n'est pas propre à la France et se retrouve dans d'autres pays, comme au Luxembourg $^{29}$ par exemple. Enfin, le droit de propriété et ses attributs sont aussi confrontés à l'exercice d'activités économiques (agricole, forestière, etc.) ou de loisirs, et sont sans cesse à la recherche d'un compromis entre différents intérêts, privés comme généraux.
27. Les documents de gestion des forêts sont les suivants : les documents d'aménagement ; les plans simples de gestion; les règlements types de gestion ; les codes des bonnes pratiques sylvicoles. 28. Articles R. 425-4 à R. 425-30 du Code de l'environnement.
29. Cour européenne des droits de l'homme, arrêt du 10 juillet 2007, Schneider c/ Luxembourg, $\mathrm{n}^{\circ} 2113 / 04$. 


\section{RÉFÉRENCES BIBLIOGRAPHIQUES}

Articles 1382 et 1383 du Code civil.

Article R. 123-2 et suivants du Code de la construction et de l'habitation.

Articles L. 362-1 et suivants, R. 362-1 et suivants, L. 422-1 et suivants, R. 422-1 et suivants, L. 425-6 et suivants, R. 425-4 et suivants, R. 425-28 et suivants, R. 426-1 et suivants du Code de l'environnement.

Articles L. 4, L. 321-1 et suivants, R. 3211 et suivants, L. 331-2 et suivants, R. 322-1 et suivants, R. 331-3 et suivants et R. 412-16 du Code forestier.

Articles L. 2213-4, L. 2215-3 et L. 2212-4 du Code général des collectivités territoriales.

Article R. 411-24 du Code de la route.

Article L. 415-1 et suivants, D. 415-1 et suivants du Code rural et de la pêche maritime.

Circulaire $\mathrm{n}^{\circ}$ DGA/SDAJ/BDEDP $\mathrm{n}^{\circ} 1 \mathrm{du}$ ministère de l'Écologie du 6 septembre 2005.

Conseil d'État (2007). 9 novembre, $\mathrm{n}^{\circ} 293644$.

Conseil d'État (2007). 9 novembre, $\mathrm{n}^{\circ} 296858$.

Conseil d'État (2008). M.H., 16 juin, $\mathrm{n}^{\circ} 296632$.

Conseil d'État (2008). Association communale de chasse agréée de Louin, 16 juin, $\mathrm{n}^{\circ} 297568$

Cour administrative d'appel de Bordeaux (2005). 14 juin, $n^{\circ}$ 01BX02337, ACCA de Bignoux et $n^{\circ}$ 01BX01733, ACCA de Liguge.

Cour d'appel de Chambéry, chambre correctionnelle, (1995). Annales de la voieries. 29 mars, n 28 avril-mai 1996, p. 4.

Cour d'appel de Paris (2004). 13 décembre, $n^{\circ} 03 / 16881$.

Cour d'appel de Dijon (5 avril 1996). Gazette du Palais. 1998, I, sommaire 392.
Cour de cassation, chambre civile (1952). Dalloz, 30 avril, $n^{\circ} 471$.

Cour de cassation, chambre criminelle (1957). Bulletin criminel, 19 février, $\mathrm{n}^{\circ} 163$, p. 277.

Cour de cassation, deuxième chambre civile (1964). Bulletin civil, II, 5 juin, ${ }^{\circ} 512$.

Cour de cassation, chambre sociale (1967). Bulletin civil, 13 avril, $\mathrm{n}^{\circ} 292$.

Cour de cassation, deuxième chambre civile (1971). Dalloz, 29 mars, sommaire 203.

Cour de cassation, deuxième chambre civile (1972). Dalloz, $1^{\text {er }}$ juin, $n^{\circ} 554$.

Cour de cassation (1974). Bulletin civil, II, 4 janvier, $n^{\circ} 6$.

Cour de cassation, deuxième chambre civile (1975). Bulletin civil, II, 15 décembre, $\mathrm{n}^{\circ} 336$.

Conseil constitutionnel (1982). Nationalisations, 16 janvier.

Cour de cassation, deuxième chambre civile (1985). Bulletin civil, II, 10 février, $\mathrm{n}^{\circ} 44$.

Cour de cassation, deuxième chambre civile (1990). 28 mars, $n^{\circ} 89-12.646$.

Cour de cassation, deuxième chambre civile (1992). 21 juillet, $\mathrm{n}^{\circ}$ 91-13.823.

Cour de cassation, deuxième chambre civile (1992). 14 octobre, $n^{\circ}$ 94-14.211.

Cour de cassation, deuxième chambre civile, (1994). JCP 95, IV, 30 novembre, n 283.

Cour de cassation, deuxième chambre civile (1997). 19 mars, $\mathrm{n}^{\circ}$ 95-14.677.

Cour de cassation, chambre criminelle (2003). 18 février, D. 2003, IR, p. 944.

Cour de cassation, deuxième chambre civile (2003). 18 septembre, $n^{\circ}$ 02-14.638.

Cour européenne des droits de l'homme (1999). Arrêt Chassagnou et autres c/ France. 29 avril, requêtes $n^{\circ} 25088 / 94$, $28331 / 95$ et $28443 / 95$

Cour européenne des droits de l'homme (2007). Arrêt M. Mme Gilbert Baudi- 
nière, requête $\mathrm{n}^{\circ}$ 25708/03 et Arrêt M. Mme René Vauzelle c/ France, requête, 6 décembre, $n^{\circ}$ 25719/03.

Cour européenne des droits de l'homme (2007). Arrêt Schneider c/ Luxembourg, 10 juillet, requête $\mathrm{n}^{\circ} 2113 / 04$.

De Montbron R. (2008). ACCA, le Conseil d'État confirme la validité du droit de retrait. La Propriété Privée Rurale, $\mathrm{n}^{\circ} 373$.

Lagier C. (2008). Note sous CEDH 6 décembre 2007, M. Mme Gilbert Baudinière, requête $\mathrm{n}^{\circ} 25708 / 03$ et M. Mme René Vauzelle c/ France, requête $\mathrm{n}^{\circ} 25719 / 03$, Droit de l'environnement, $\mathrm{n}^{\circ} 158$.

Lagier C. (2008). Droit de la chasse : chronique de l'année 2007. Gazette du Palais, $\mathrm{n}^{\circ} 228$ à 232.

Havet P. (2007). Les leçons de l'histoire, nature, chasse et société. L'Harmattan, La chasse en quête de sens, vol. 1.

Minne H. (2007). Droit de chasser et droit de chasse. La Propriété Privée Rurale, $\mathrm{n}^{\circ} 367$.
Privat C. (2007). Vers un nouveau partage du milieu naturel ? Réflexions sur les conséquences liées à la remise en cause des associations communales de chasse agréées. Revue Française de droit administratif, mars-avril, p. 354.

Venner D. (2007). La chasse, dernier refuge du sauvage? Privat.

Vigier J. (2007). Écologie, chasse, propriété, Europe et loi : un quarteron pour une quadrature. Mélanges en l'honneur de Michel Prieur, « Pour un droit commun de l'environnement », Dalloz.

Peignot B. (2006). Le droit de chasse : attribut ou démembrement du droit de propriété ? Agriculteurs de France, $\mathrm{n}^{\circ} 165$, septembre-octobre, p. 24.

Patriat F. (2000). Propositions pour une chasse responsable et apaisée. Rapport au Premier ministre, La Documentation Française.

Tribunal civil (1862). Melun, 21 février. Tribunal des conflits (1999). 3 mai, $n^{\circ} 3114$, SCEA de Courbevoie. 